\title{
Bandgap Tailoring and Synchronous Microdevices Patterning of Graphene Oxides
}

\author{
Li Guo, $^{\dagger}$ Rui-Qiang Shao, ${ }^{\dagger}$ Yong-Lai Zhang, ${ }^{* \dagger}$ Hao-Bo Jiang, $^{\dagger}$ Xian-Bin Li, ${ }^{\dagger}$ Sheng-Yi Xie, ${ }^{\dagger}$ Bin-Bin Xu, ${ }^{\dagger}$ \\ Qi-Dai Chen, ${ }^{\dagger}$ Jun-Feng Song, ${ }^{\dagger}$ and Hong-Bo Sun ${ }^{*, \dagger, \dagger}$
}

${ }^{\dagger}$ State Key Laboratory on Integrated Optoelectronics, College of Electronic Science and Engineering, Jilin University, 2699 Qianjin Street, Changchun 130012, P. R. China

${ }^{\ddagger}$ College of Physics, Jilin University, 119 Jiefang Road, Changchun 130023, P. R. China

\section{Supporting Information}

ABSTRACT: Reported here is femtosecond laser mediated bandgap tailoring of graphene oxides (GOs) for direct fabrication of graphene-based microdevices. When femtosecond laser pulses were used to reduce and pattern GO, oxygen contents in the reduced region could be modulated by varying the laser power. In this way, the bandgap of reduced GO was precisely modulated from 2.4 to $0.9 \mathrm{eV}$ by tuning the femtosecond laser power from 0 to $23 \mathrm{~mW}$. Through the first-principle study, the essence of GO bandgap tailoring is proved to be femtosecond laser reduction induced oxygen-content modulation. As representative illustrations, bottom-gate graphene FETs were fabricated in situ by using femtosecond laser reduced GO as

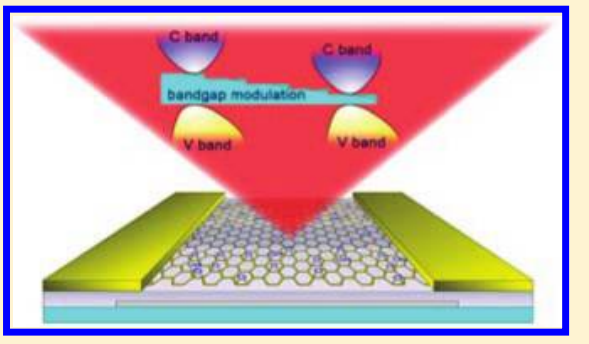
the channel material, and an optimized room temperature on-off ratio of 56 is obtained. The controlled reduction of GO by femtosecond laser contributes great potential for bandgap tailoring and microdevices patterning of graphene toward future electronics.

\section{INTRODUCTION}

In recent years, graphene has triggered enormous research interest due to its great potential in future electronics, ${ }^{1-5}$ such as flexible e-paper. However, open problems such as scalable preparation, bandgap tailoring, devices fabrication, and postintegration are still challenging. For instance, graphene prepared by epitaxial growth is not only limited in massproduction, but also undergoes a complex substrate transfer process, which brings considerable difficulties to its further applications. More importantly, graphene is a zero-bandgap semiconductor; its performance in field effect transistors (FETs), ${ }^{6,7}$ microsensors, ${ }^{8-12}$ photodiodes ${ }^{13}$ and lasers, ${ }^{14}$ was significantly limited due to the absence of bandgap. ${ }^{5}$ Therefore, tailoring electronic band structures becomes a hot topic in the current investigation on graphene-based microdevices. Typically, nanostructuring of graphene has been proved an effective solution to open its bandgap. ${ }^{15,16}$ When the size of graphene was structured into mesoscopic range, usually from several to tens of nanometers, energy bandgap would form due to the quantum confinement of electrons. However, the modulation range of graphene bandgaps was quite limited because it is very difficult to reduce its lateral dimensions into the sub-10 nm range. As an alternative choice, similar to the case of carbon nanotubes (CNTs), ${ }^{17}$ molecular doping is also used for graphene bandgap tailoring. ${ }^{18,19}$ By various chemical grafting or physical adsorption of guest molecules, the band structure of graphene could be modulated in a certain range. However, these methods usually suffer from poor stability or controllability. Therefore, in order to promote the development of graphene nanodevices, a versatile nanotechnology for both processing and bandgap tailoring of graphene is highly desired.

From the practical point of view, graphene oxides (GOs) have distinct advantages of (i) large-scale preparation, (ii) solution processing compatibility, and (iii) tractable modification. ${ }^{20}$ Essentially, the formation of bandgaps usually depends on defects, so GO, which has plenty of natural oxygen-defects, is undoubtedly considered as a preferred candidate for bandgap tailoring. ${ }^{21-23}$ The fact that laser irradiation could effectively reduce $\mathrm{GO}^{24,25}$ imparts the feasibility for further modulation of its electronic band structure. In addition, laser processing allows high accuracy and mask-free micronanopatterning, which contributes supplementary advantages for microdevice fabrication and integration.

In this work, we show a femtosecond laser mediated bandgap tailoring of graphene oxides (GO) for flexible fabrication of graphene-based nanodevices. Bandgaps of reduced GO could be precisely modulated from 2.4 to $0.9 \mathrm{eV}$ by controlling the laser power. First-principle study confirms that the residual oxygen in reduced GO was essential for bandgap formation. As representative illustrations, bottom-gate graphene FETs were fabricated in situ by postintegration of the laser reduced GO (LR-GO) channel between prepatterned source/drain electrodes. The femtosecond laser processing of GO contributes great advantages for flexible fabrication of graphene-based nanodevices in future electronics.

Received: October 13, 2011

Revised: January 6, 2012

Published: January 9, 2012 


\section{EXPERIMENTAL METHODS}

2.1. Femtosecond Laser Reduction of GO Films. The graphene oxide was produced via Hummers method from natural graphite (Aldrich, $<150 \mu \mathrm{m}$ ). The GO films were prepared by spin-coating GO solution on quartz substrate at $2000 \mathrm{rpm}$ for $20 \mathrm{~s}$, dried at $95{ }^{\circ} \mathrm{C}$ for $1 \mathrm{~h}$, and cooled to room temperature. Pulses from a femtosecond laser oscillator of 780 $\mathrm{nm}$ central wavelength, $120 \mathrm{fs}$ pulse duration, and $80 \mathrm{MHz}$ repetition rate, were tightly focused by a $100 \times$ oil immersion objective lens with a high numerical aperture (NA) of 1.35 into the GO film. The focal spot of the laser beam was scanned laterally by steering a two-galvano-mirror set and was vertically moved along the optical axis by a piezo stage; $8-23 \mathrm{~mW}$ laser power measured before the objective lens, $600 \mu$ s exposure duration of each voxel, and $100 \mathrm{~nm}$ scanning step length were adopted. Then, the femtosecond laser directly wrote on the GO film according to preprogrammed patterns. The femtosecond laser was generated by Tsunami, Spectra-Physics lasers (model, 3960-X1BB s/n 2617; ccd, AMSTAR, B/W; video ccd, CAMERA).

2.2. Fabrication and Measurement of GO FETs. The ITO gate electrode was sputtered onto the substrates through a shadow mask. To obtain a gate dielectric layer, poly(methyl methacrylate)(PMMA) chloroform solution $(0.1 \mathrm{~g} / \mathrm{mL})$ was spin-coated on substrates at $3000 \mathrm{rpm}$ and baked at $95{ }^{\circ} \mathrm{C}$ for $15 \mathrm{~min}$. The thickness of PMMA is measured to be $200 \mathrm{~nm}$. GO films were spin-coated on PMMA layer and annealed at 95 ${ }^{\circ} \mathrm{C}$ for $1 \mathrm{~h}$. Au source and drain electrodes were vaporized onto the GO film under vacuum by using a shadow mask. In order to modulate the active layer, GO film was then reduced and patternd into a $30 \mu \mathrm{m}$ width belt between the electrodes (distance, $20 \mu \mathrm{m}$ ) for test.

2.3. Characterization. The UV-vis/NIR diffuse reflectance spectra of the GO film was measured from the UVvisible diffuse reflection spectroscopy (UV-3600, SHIMADZU, Japan) with a $\mathrm{BaSO}_{4}$ coated integration sphere at room temperature. For the measurement of bandgap, a $2 \times 2 \mathrm{~mm}^{2}$ GO square was reduced by a femtosecond laser with variable output power. X-ray photoelectron spectroscopy (XPS) was performed using an ESCALAB 250 spectrometer. Atomic force microscopy (AFM) images were measured with Digital Instruments NanoScope IIIa. Optical microscope images were obtained from a Motic BE400 microscope. Current-voltage curves of FET were measured from a Keithley SCS 4200 semiconductor characterization system.

\section{RESULTS AND DISCUSSION}

In order to gradually remove the oxygen groups on GO sheets and further modulate their bandgaps in a certain range, femtosecond laser pulse with adjustable output power was used for the reduction. Because of the ultra fast pulse duration (120 fs) of our femtosecond laser, here, GO is allowed to be reduced in air without total ablation. In our experiments, by carefully tuning the laser power, the reduction degree of GO could be controlled in the irradiated region. Figure 1 shows the C1s XPS spectra of pristine GO and laser-reduced GO (LR-GO) samples. The three peaks at 284.6, 286.6, and $288.5 \mathrm{eV}$ are attributed to $\mathrm{C}-\mathrm{C}$ (nonoxygenated ring carbon), $\mathrm{C}-\mathrm{O}$ (hydroxyl and epoxy carbon), and $\mathrm{C}=\mathrm{O}$ (carbonyl), respectively. Obviously, the as-prepared GO is very rich in oxygen; the content of carbon not bonded to oxygen is only $38 \%$. After femtosecond laser reduction $(10 \mathrm{~mW})$, the $\mathrm{C}-\mathrm{C}$

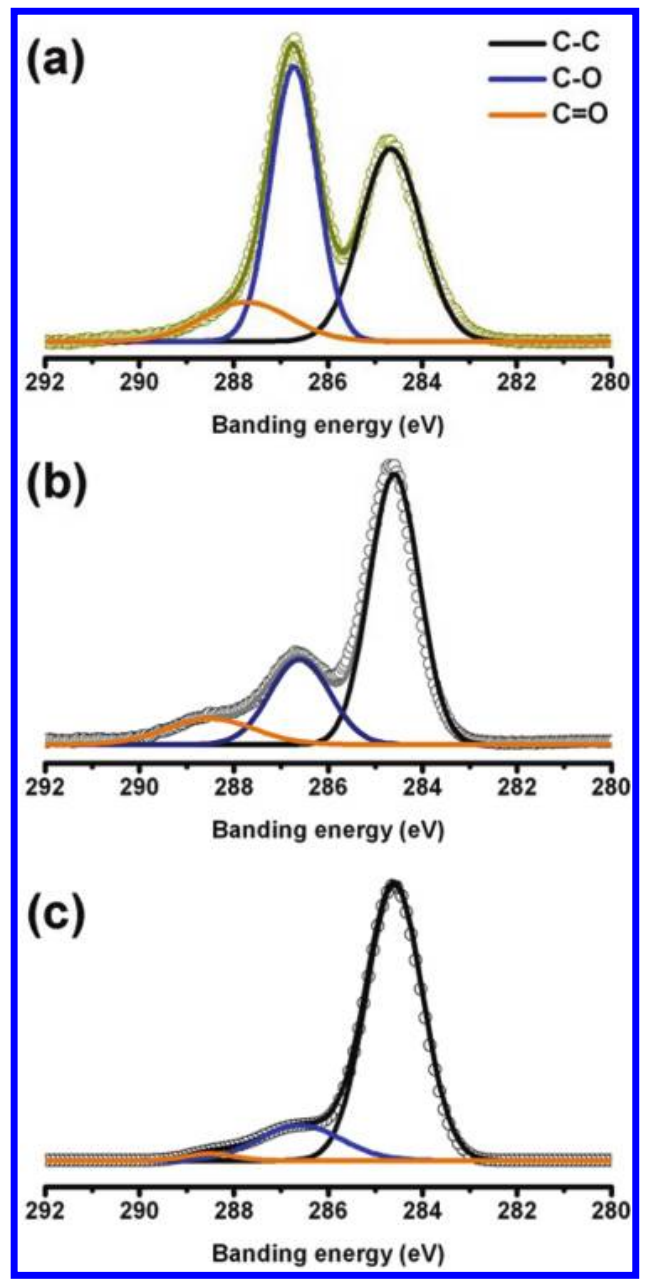

Figure 1. C1s XPS spectra of pristine GO (a), LR-GO (b, $10 \mathrm{~mW})$, and LR-GO (c, $23 \mathrm{~mW})$. The C1s spectra of GO and LR-GOs are deconvoluted into three peaks at 284.6, 286.6, and $288.5 \mathrm{eV}$, corresponding to $\mathrm{C}-\mathrm{C}, \mathrm{C}-\mathrm{O}$, and $\mathrm{C}=\mathrm{O}$, respectively.

percentage increases to $65 \%$, indicating the partial removal of oxygen. When the laser power is further increased to $23 \mathrm{~mW}$, the content of carbon not bonded to oxygen is as high as $83 \%$, meaning that a majority of oxygen-containing groups are removed. Survey X-ray photoelectron spectra of the three samples confirm that oxygen content decreases with the increase of femtosecond laser power (Figure S1, Supporting Information), indicating the controllable removal of oxygen.

The mechanism of the oxygen removing in our work is mainly ascribed to electronic excitation effect and the electronhole $(\mathrm{e}-\mathrm{h})$ recombination induced thermal effect. During the femtosecond laser reduction, the electronic excitation effect is significant in the first several picoseconds. In this condition, electrons could be excited from bonding states to antibonding states. The excitation could significantly weaken $\mathrm{C}-\mathrm{O}$ electronic bonding near the top of the valence band and therefore lead to an immediate oxygen removal. After sufficient electron-hole $(\mathrm{e}-\mathrm{h})$ recombination (i.e., $>\sim 100 \mathrm{ps}$ ), the normal heat reduction on GO is dominated. Beside the laser power, the irradiation time also shows similar influence on the oxygen content control. However, as compared with laser power, the effect is less obvious, and the modulation range is relatively small. So in this work, we fix the exposure time at 600 $\mu \mathrm{s}$ and change the laser power for further modulation. 
Raman spectra of pristine GO and LR-GO films were measured to evaluate the structural change before and after femtosecond laser reduction. As shown in Figure S2, Supporting Information, the $I_{\mathrm{D}} / I_{\mathrm{G}}$ ratios of GO, LR-GO (10 $\mathrm{mW})$, and LR-GO $(23 \mathrm{~mW})$ were $0.98,0.90$, and 0.84 , respectively. A decrease of the $I_{\mathrm{D}} / I_{\mathrm{G}}$ ratio is perhaps an indication of graphitization. The Raman map of the $I_{\mathrm{D}} / I_{\mathrm{G}}$ ratio shows the pattern of the LR-GO due to the difference in the $D /$ $G$ intensity. Furthermore, the $I_{\mathrm{D}} / I_{\mathrm{G}}$ ratio of the LR-GO region was very uniform, indicating the homogeneous property. Note that the structure of GO is very complicated, and the oxygen distribution is not homogeneous at atom scale, concerns of homogeneity may arise in view of device fabrication. However, the LR-GO that shows homogeneity at nanoscale is considered to be homogeneous in various electronic devices.

The bandgap of the resultant LR-GO and pristine GO is measured by diffuse reflectance spectra (DRS). Typically, an overall $2 \times 2 \mathrm{~mm}^{2}$ GO film was reduced by a femtosecond laser with different power for the measurement. AFM and EFM images show that the LR-GO region has smooth surface morphology and uniform surface potentials, respectively (Figure S3, Supporting Information). Note that a different reduction degree would cause different surface morphology and conductivity; $^{24}$ this uniformity indicates the homogeneous property of the laser reduced GO. As reported elsewhere, ${ }^{21,26}$ the band gap of GO can be deduced from the UV-visible DRS in which the Kubelka-Munk function $F(R)$ was used to convert the reflectance of the sample $\left(R_{\mathrm{s}}\right)$, normalized by the reflectance of the reference $\left(R_{\mathrm{r}}\right)$, into an equivalent absorption spectrum with the following equations:

$$
\begin{aligned}
& R=\frac{R_{\mathrm{s}}}{R_{\mathrm{r}}} \\
& F(R)=\frac{(1-R)^{2}}{2 R}=\frac{\alpha}{S} \\
& \alpha \propto \frac{\left(h \nu-E_{0}\right)^{n}}{h \nu}
\end{aligned}
$$

where $h v$ is the energy of the incident photon, $\alpha$ is the absorption coefficient, $S$ is the scattering coefficient, and $E_{0}$ is the optical absorption edge energy. The exponent, $n$, depends on the type of optical transition caused by the photon absorption. In this study, $n$ was chosen for the best fit to $1 / 2$, indicating a direct-allowed optical transition since the Lowest Unoccupied Molecular Orbital (LUMO) and Highest Occupied Molecular Orbital (HOMO) meet at the $K$ point for pristine graphite. ${ }^{27} \mathrm{BaSO}_{4}$ was used as the reference sample. Figure $2 \mathrm{a}$ shows the square of the Kubelka-Munk function multiplied by the photon energy as a function of the photon energy. The abscissa of the linear fit of the absorption edges indicates the bandgap of the GO. Figure $2 \mathrm{~b}$ shows the dependence of the bandgap on the reduction laser power. Notably, after FS laser reduction, the bandgap decreases from $2.4 \mathrm{eV}$ for the pristine GO to $0.9 \mathrm{eV}$ for the LR-GO $(23 \mathrm{~mW})$. These results indicate that the band gap of laser reduced GO can be tuned easily in a certain range by changing FS laser power.

To get further insight into the origin of GO bandgap tailoring in our laser reduction experiments, the first-principle study was adopted for this investigation. Note that the atomic structure for GO is still uncertain currently. ${ }^{28-30}$ Yet, it is

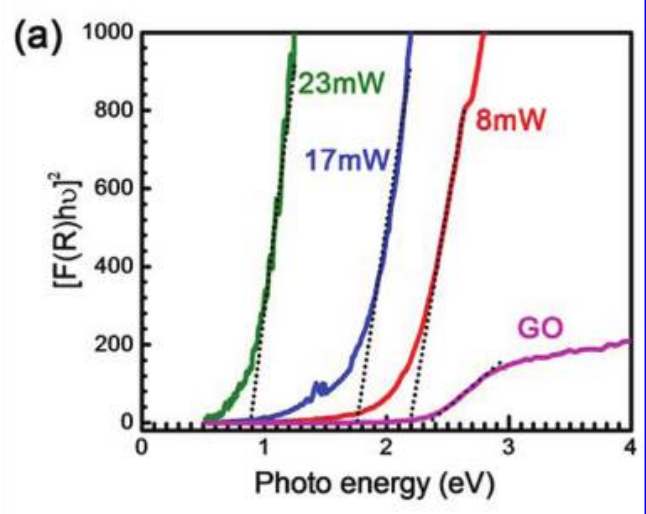

(b)

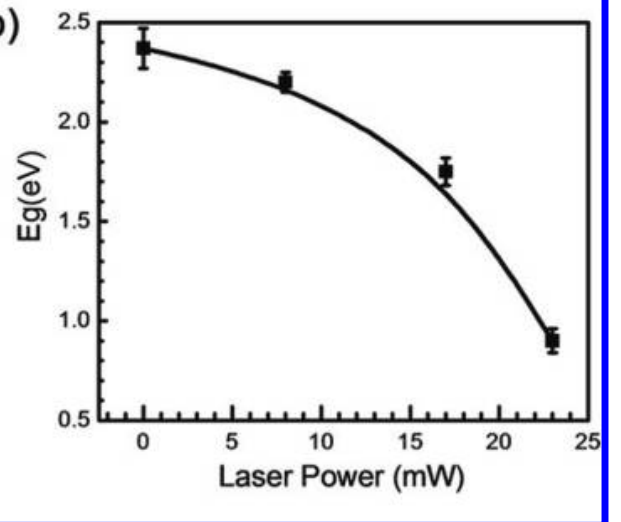

Figure 2. (a) Absorption spectra related to the Kubelka-Munk function. (b) Dependence of LR-GO bandgap on the reduction laser power.

generally accepted that oxygen of GO is mainly in the form of epoxy and hydroxyl groups. ${ }^{31,32}$ Here, we simplify the GO model with only epoxy radical coverage on the same side of the carbon sheet instead of complicated discussion with different radicals and positions (Figure 3a). In the calculation, the variation of bandgap with 0 to $100 \%$ oxygen coverage in GO (equal to $n_{\mathrm{O}} / n_{\mathrm{C}}=0 / 32-16 / 32$ ) are in the range of 0 to 2.74 $\mathrm{eV}$ (Figure $3 \mathrm{~b}$ ). Here, the absolute bandgaps are somewhat deviated from our experimental results due to the simplification of the GO model. However, the similar tendency is observed, that is, with the decrease of oxygen content, the bandgap becomes smaller. This directly lent a support that the femtosecond laser manipulates GO bandgap through oxygen content control. Figure $3 \mathrm{c}$,d show the p-orbit DOS for different oxygen coverage and the scheme for the band gap formation process. It is well-known that the zero bandgap of graphene comes from the p-bonding band and the p-antibonding band. ${ }^{33}$ Because of the larger electronegativity, oxygen would strongly attract electrons from the carbon sheet. With the oxygen coverage increasing, the $\mathrm{p}$ electrons of the $\mathrm{O}$ atom would bind with the $\mathrm{p}$ electrons from graphene, which leads to the GO valence band being energetically lower, and oppositely the conduction band higher. As a result of the controllable oxygen contents, bandgaps were modulated in a certain range (Figure 3d).

The femtosecond laser mediated bandgap modulation of GO imparts great advantages to in situ fabrication and integration of graphene-based microdevices. Take field effect transistors (FET) as an example; conventional graphene FETs are usually fabricated by postpatterning of source and drain electrodes to 

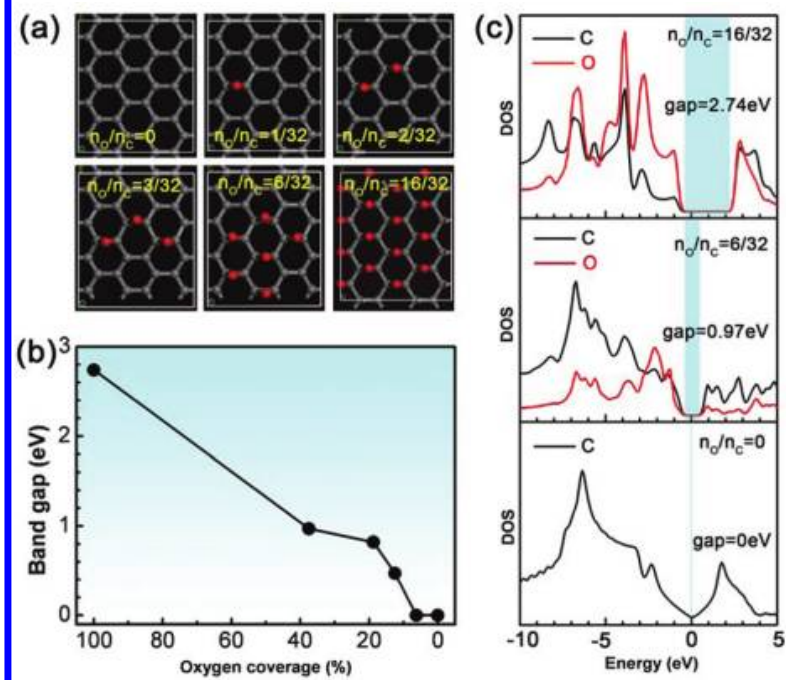

(d)
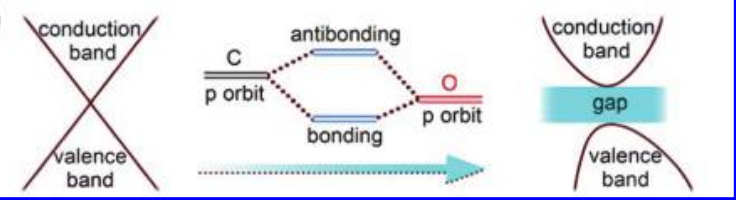

Figure 3. (a) Structures of GO with different oxygen to carbon atoms ratio $\left(n_{\mathrm{O}} / n_{\mathrm{C}}\right)$; gray and red balls are $\mathrm{C}$ and $\mathrm{O}$ atoms, respectively. (b) The calculated GO bandgaps with respect to the different oxygen coverage. (c) The p-orbit DOS for $\mathrm{C}$ and $\mathrm{O}$ atom for the oxygen coverage $0 \%\left(n_{\mathrm{O}} / n_{\mathrm{C}}=0 / 32\right), 37.5 \%\left(n_{\mathrm{O}} / n_{\mathrm{C}}=6 / 32\right)$, and $100 \%\left(n_{\mathrm{O}} /\right.$ $n_{\mathrm{C}}=16 / 32$ ). The valence band maximum is set to 0 . (d) Scheme for the bandgap formation process of GO.

connect the graphene channel including thermal/chemical reduced GO or substrate-transferred graphene sheet. Combined with femtosecond laser direct writing, our LR-GO channel could be created freely between two precoated electrodes, which hold great promise for flexible integration of graphene-based microdevices. As shown in Figure 4a, a bottom-gate FET was constructed with ITO as the gate and PMMA as the gate dielectric layer. In the whole fabrication process, the channel (here, LR-GO) with controllable bandgap could be created at desired position in the last step, indicating the unique feature of flexible fabrication. Moreover, the powerful femtosecond laser processing allows flexible fabrication of micronanopatterns with high resolution; ${ }^{25,34-38}$ therefore, the channel could be shaped into various shapes and patterns. As shown in Figures $4 \mathrm{~b}-\mathrm{d}$, parallel microlines with different width, sinusoid, and hexagon grid were directly patterned between electrodes. Notably, the GO film under the $\mathrm{Au}$ electrodes could also be reduced (Figure $4 \mathrm{~b}-\mathrm{d}$ ), so a better ohmic contact between source/drain electrodes and the graphene channel is expected compared with conventional approaches. The current-voltage curves of the above-three channels are presented in Figure 4e, confirming the good ohmic contact and conductivity of the patterned LR-GO. Note that adjustable resistance was observed due to the different reduction area of the GO channel; the LR-GO FETs could be flexibly tuned with suitable working current.

In the test of our FETs, GO was simply patterned into a $20 \times$ $30 \mu \mathrm{m}$ belt as channel materials between source and drain electrodes. Figure 5 shows the output characteristics of four FETs, which are fabricated with GO and LR-GOs (reduced by different laser powers) as channels. Notably, a GO film without

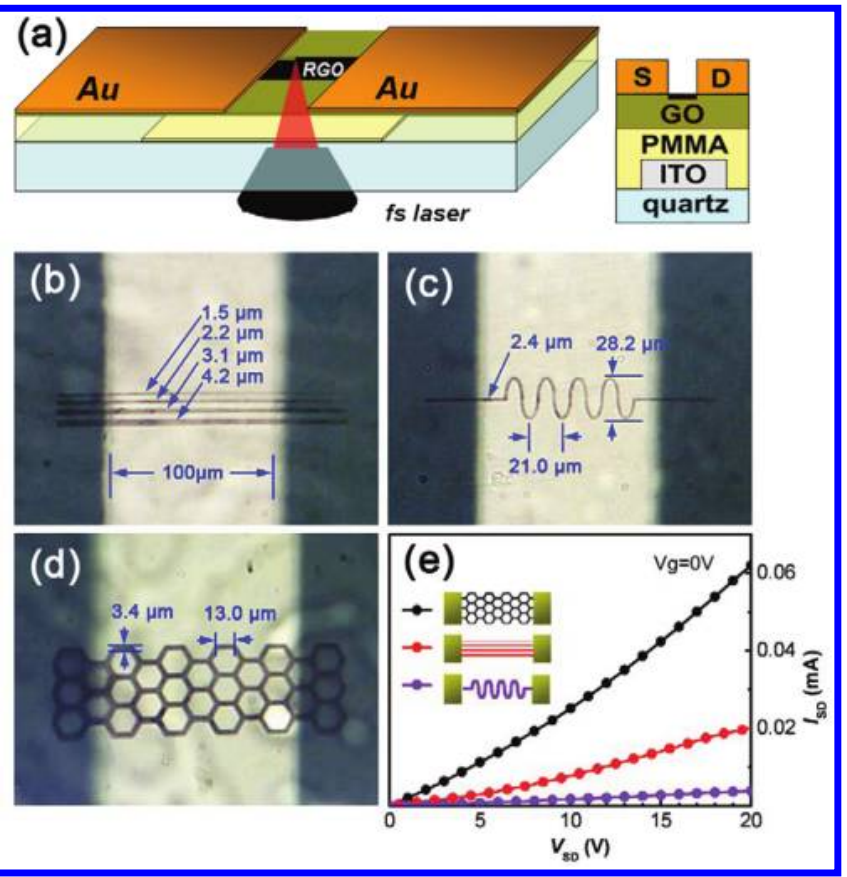

Figure 4. (a) Scheme of the bottom-gate GO FET. Optical microscopic images of (b) parallel microlines with different width, (c) sinusoid, and (d) hexagon grid was patterned between source and drain electrodes as a channel. (e) I-V curves of the above three channels with $V_{\mathrm{g}}=0$.

reduction shows no gate effect (Figure 5a) due to its wide bandgap and the high hole-injection barrier between the $\mathrm{Au}$ electrodes and GO. The very small $S / D$ current indicates that the carrier concentration is very low, so GO is insulating to some extent. In this case, the pristine GO film could be considered as a part of the substrate for the later devices. When femtosecond laser was used to modulate the bandgap of GO film between source and drain electrodes, a LR-GO channel with adjustable bandgap was created. As shown in Figure $5 \mathrm{~b}$, conductivity of the channel with a bandgap of $2.1 \mathrm{eV}$ (LR-GO, $10 \mathrm{~mW}$ ) increased under negative gating conditions due to the residual oxygen functionality induced hole doping, indicating its p-type behavior. ${ }^{26}$ The device shows a $I_{\text {on }} / I_{\text {off }}$ ratio of 56 with an estimated hole mobility of $0.19 \mathrm{~cm}^{2} \mathrm{~V}^{-1} \mathrm{~s}^{-1}$, which is estimated from the following equation:

$$
\mu=\left(L / W C_{\mathrm{ox}} V_{\mathrm{d}}\right) /\left(\Delta I_{\mathrm{d}} / \Delta V_{\mathrm{g}}\right)
$$

where $C_{\mathrm{ox}}=\varepsilon_{0} \varepsilon_{\mathrm{PMMA}} / t_{\mathrm{ox}}$ is capacitance per unit area of the gate insulator; $\varepsilon_{0}$ is vacuum permittivity $\left(8.85 \times 10^{-12} \mathrm{~F} / \mathrm{m}\right) ; \varepsilon_{\text {PMMA }}$ is the relative dielectric constant of PMMA (3.0); $t_{\mathrm{ox}}$ is the thickness of PMMA (200 nm); $L$ and $W$ are channel length (20 $\mu \mathrm{m})$ and width $(30 \mu \mathrm{m})$, respectively. When we increase the laser power to $16 \mathrm{~mW}$, the bandgap of the channel is reduced to $1.7 \mathrm{eV}$ and a $I_{\mathrm{on}} / I_{\text {off }}$ ratio of 6.5 with an estimated hole mobility of $1.17 \mathrm{~cm}^{2} \mathrm{~V}^{-1} \mathrm{~s}^{-1}$ was observed (Figure 5c). With the decrease of bandgap, carrier concentration increases at room temperature. The decrease of on-off ratio is explained as the increases of carrier concentration, which covered the modulation of gate voltage partly. The mobility has a great relationship with reduction level since the scattering of carriers is caused by the oxygen defects. As a result, an increase of mobility is observed when the oxygen groups decrease. A channel reduced by laser power of $23 \mathrm{~mW}$ shows no gate bias modulation, indicating its metallic behavior (Figure 5d). These 

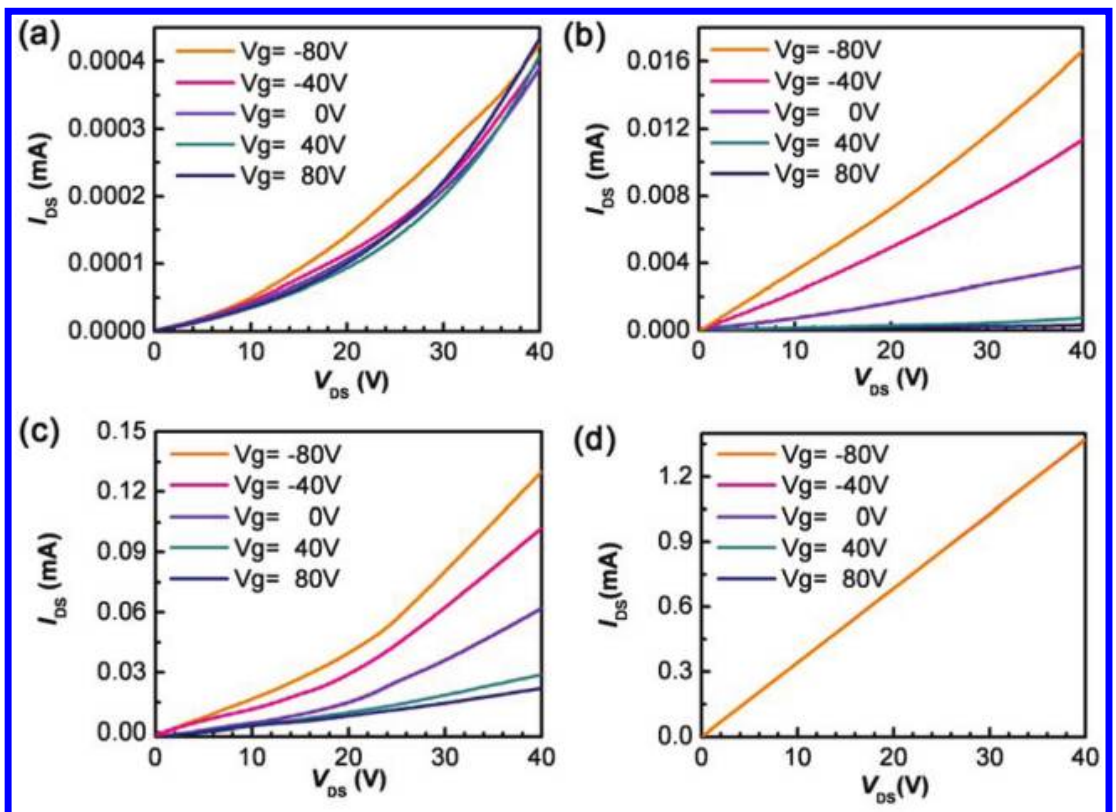

Figure 5. Output characteristics of four FETs with (a) GO, (b) LR-GO (10 mW), (c) LR-GO (17 mW), and (d) LR-GO (23 mW) as channels, respectively. The gate voltage is in the range of $-80-80 \mathrm{~V}$.

results reveal that the performance of GO FET could be optimized by modulating the channel bandgap in a controlled fashion. In the analysis of our FETs, we did not consider the effect of the intrinsic GO region since it performs as a substrate due to its insulation property. Notably, this selected reduction for device fabrication is not a special case; similar device structure could be found in the traditional doping of intrinsic silicon in IC technology. The difference is that the laser direct writing process can achieve regulation of the semiconductor in a mask-free manner. This direct patterning and synchronous bandgap tailoring of GO might find broad applications in soft drive circuits for OLED display in the near future.

\section{CONCLUSIONS}

In conclusion, a femtosecond laser modulation of GO bandgap was successfully developed toward flexible fabricaton of graphene-based microdevices. After irradiation by a femtosecond laser with different output powers, LR-GOs with variable oxygen content were obtained. Moreover, the bandgap of GO was modulated in the range of 2.4 to $0.9 \mathrm{eV}$ by tuning the femtosecond laser power from 0 to $23 \mathrm{~mW}$. Through the first-principle study, the origin of GO bandgap tailoring is explained as femtosecond laser reduction induced oxygencontent control. As a representative test, bottom-gate graphene FETs were successfully fabricated, and an optimized room temperature on-off ratio of 56 is obtained by tuning the laser power. The novel femtosecond laser modulation of GO makes great contributions to flexible fabrication of graphene-based microdevices, and thus, it holds great promise for future nanoelectronics.

\section{ASSOCIATED CONTENT}

\section{S Supporting Information}

Survey X-ray photoelectron spectra, Raman spectra of GO and LR-GOs, Optical microscopic image of an LR-GO square, Raman maps of $\mathrm{D} / \mathrm{G}$ intensity ratio, AFM image and EFM image of laser reduced RGO and pristine GO, and method for first-principle study. This material is available free of charge via the Internet at http://pubs.acs.org.

\section{AUTHOR INFORMATION}

\section{Corresponding Author}

*Tel/Fax: +86 (431) 8516-8281. E-mail: yonglaizhang@jlu. edu.cn (Y.-L.Z.); hbsun@jlu.edu.cn (H.-B.S.). Homepage: http://lasun-jlu.cn/.

\section{ACKNOWLEDGMENTS}

We acknowledge the financial support from 973 program Grant No. 2011CB013005, NSFC under grant nos. 61008104, 90923037, 60978062, and 11104109, the China Postdoctoral Science Foundation under grant no. 20110490156, the Hong Kong Scholar Program XJ2011014, and the TNList crossdiscipline foundation. We also thank the High Performance Computing Center (HPCC) of Jilin University for calculation resources.

\section{REFERENCES}

(1) Novoselov, K. S.; Geim, A. K.; Morozov, S. V.; Jiang, D.; Zhang, Y.; Dubonos, S. V.; Grigorieva, I. V.; Firsov, A. A. Science 2004, 306, 666-669.

(2) Zhang, Y.; Tan, Y. W.; Stormer, H. L.; Kim, P. Nature 2005, 438, 201-204.

(3) Novoselov, K. S.; Geim, A. K.; Morozov, S. V.; Jiang, D.; Katsnelson, M. I.; Grigorieva, I. V.; Dubonos, S. V.; Firsov, A. A. Nature 2005, 438, 197-200.

(4) Berger, C.; Song, Z.; Li, X.; Wu, X.; Brown, N.; Naud, C.; Mayou, D.; Li, T.; Hass, J.; Marchenkov, A. N.; Conrad, E. H.; First, P. N.; de Heer, W. A. Science 2006, 312, 1191-1196.

(5) Geim, A. K.; Novoselov, K. S. Nat. Mater. 2007, 6, 183-191.

(6) Oostinga, J. B.; Heersche, H. B.; Liu, X. L.; Morpurgo, A. F.; Vandersypen, L. M. K. Nat. Mater. 2008, 7, 151-157.

(7) Liao, L.; Bai, J. W.; Lin, Y. C.; Qu, Y. Q.; Huang, Y.; Duan, X. F. Adv. Mater. 2010, 22, 1941-1945.

(8) Zhang, Y.; Tang, Z. R.; Fu, X.; Xu, Y. J. ACS Nano 2010, 4, 7303-7314.

(9) Ang, P. K.; Chen, W.; Wee, A. T. S.; Loh, K. P. J. Am. Chem. Soc. 2008, 130, 14392-14393. 
(10) Ji, Q. M.; Honma, I.; Paek, S. M.; Akada, M.; Hill, J. P.; Vinu, A.;

Ariga, K. Angew. Chem., Int. Ed. 2010, 49, 9737-9739.

(11) Johnson, J. L.; Behnam, A.; Pearton, S. J.; Ural, A. Adv. Mater. 2010, 22, 4877-4880.

(12) Ohno, Y.; Maehashi, K.; Matsumoto, K. J. Am. Chem. Soc. 2010, 132, 18012-18013.

(13) Mueller, T.; Xia, F. N.; Avouris, P. Nat. Photonics 2010, 4, 297301.

(14) Sun, Z. P.; Hasan, T.; Torrisi, F.; Popa, D.; Privitera, G.; Wang, F. Q.; Bonaccorso, F.; Basko, D. M.; Ferrari, A. C. ACS Nano 2010, 4, $803-810$.

(15) Barone, V.; Hod, O.; Scuseria, G. E. Nano Lett. 2006, 6, 27482754.

(16) Son, Y. W.; Cohen, M. L.; Louie, S. G. Phys. Rev. Lett. 2006, 97, 216803.

(17) Nakamura, S.; Ohishi, M.; Shiraishia, M. Appl. Phys. Lett. 2006, 89,013112 .

(18) Wei, D. C.; Liu, Y. Q.; Wang, Y.; Zhang, H. L.; Huang, L. P.; Yu, G. Nano Lett. 2009, 9, 1752-1758.

(19) Berashevich, J.; Chakraborty, T. Phys. Rev. B 2009, 80, 033404.

(20) Stankovich, S.; Dikin, D. A.; Dommett, G. H. B.; Kohlhaas, K. M.; Zimney, E. J.; Stach, E. A.; Piner, R. D.; Nguyen, S. T.; Ruoff, R. S. Nature 2006, 442, 282-286.

(21) Jeong, H. K.; Jin, M. H.; So, K. P.; Lim, S. C.; Lee, Y. H. J. Phys. D: Appl. Phys. 2009, 42, 065418.

(22) Chang, H. X.; Sun, Z. H.; Yuan, Q. H.; Ding, F.; Tao, X. M.; Yan, F.; Zheng, Z. J. Adv. Mater. 2010, 22, 4872-4876.

(23) Luo, Z.; Vora, P. M.; Mele, E. J.; Johnson, A. T. C.; Kikkawa, J. M. Appl. Phys. Lett. 2009, 94, 111909-111911.

(24) Zhang, Y. L.; Guo, L.; Wei, S.; He, Y. Y.; Xia, H.; Chen, Q. D.; Sun, H. B.; Xiao, F. S. Nano Today 2010, 5, 15-20.

(25) Zhang, Y. L.; Chen, Q. D.; Xia, H.; Sun, H. B. Nano Today 2010, 5, 435-448.

(26) Khan, S. U. M.; Al-Shahry, M.; Ingler, W. B. Science 2002, 297, 2243-2245.

(27) McClure, J. W. Phys. Rev. 1957, 108, 612-618.

(28) Szabó, T.; Berkesi, O.; Forgó, P.; Josepovits, K.; Sanakis, Y.; Petridis, D.; Dékány, I. Chem. Mater. 2006, 18, 2740-2749.

(29) Wang, L.; Sun, Y. Y.; Lee, K.; West, D.; Chen, Z. F.; Zhao, J. J.; Zhang, S. B. Phys. Rev. B 2010, 82, 161406(R).

(30) Yan, J.-A.; Xian, L.; Chou, M. Y. Phys. Rev. Lett. 2009, 103, 086802.

(31) Cai, W. W.; Piner, R. D.; Stadermann, F. J.; Park, S.; Shaibat, M. A.; Ishii, Y.; Yang, D. X.; Velamakanni, A.; An, S. J.; Stoller, M.; An, J.; Chen, D. M.; Ruoff, R. S. Science 2008, 321, 1815-1817.

(32) Gao, W.; Alemany, L. B.; Ci, L.; Ajayan, P. M. Nat. Chem. 2009, $1,403-408$.

(33) Castro Neto, A. H.; Guinea, F.; Novoselov, K. S.; Geim, A. K. Rev. Mod. Phys. 2009, 81, 109-162.

(34) Kawata, S.; Sun, H. B.; Tanaka, T.; Takada, K. Nature 2001, 412, 697-698.

(35) Xia, H.; Wang, J.; Tian, Y.; Chen, Q. D.; Du, X. B.; Zhang, Y. L.; He, Y.; Sun, H. B. Adv. Mater. 2010, 22, 3204-3207.

(36) Tian, Y.; Zhang, Y. L.; Ku, J. F.; He, Y.; Xu, B. B.; Chen, Q. D.; Xia, H.; Sun, H. B. Lab Chip 2010, 10, 2902-2095.

(37) Lee, K. S.; Yang, D. Y.; Park, S. H.; Kim, R. H. Polym. Adv. Technol. 2006, 17, 72-82.

(38) Zhou, Y. S.; Xiong, W.; Gao, Y.; Mahjouri-Samani, M.; Mitchell, M.; Jiang, L.; Lu, Y. F. Nanotechnology 2010, 21, 315601. 